\title{
CONTINUATORI BALCANICO-DANUBIANI DEL VENETO "BALOTA"
}

E' merito di László Hadrovics di avere impostato una numerosa serie di ricerche etimologiche con un metodo originale che ha dato ampi frutti, fondato sullo studio globale di tanti dati, $\mathrm{ma}$ in primo luogo saldamente ancorato al minuzioso esame delle fonti e dei motivi storici e culturali che hanno occasionato i prestiti. I suoi contributi alla storia e all'etimologia di varie parole ungheresi costituiscono autentiche piccole monografie concentrate nel settore del lessico giudicato di norma "di etimologia difficile" nei dizionari specializzati; essi sono ora in buona parte riuniti in due volumetti: Jövevényszó-vizsǵalatok [Indagini sui prestiti] del $1965^{1}$ e Szavak és szólások [Parole ed espressioni] del 1975 2 . In questa seconda miscellanea, nel capitolo quinto dedicato ai "prestiti italiani" (olasz jövevényszók, alle pp. 78-94) l'A. ha l'occasione di occuparsi della voce ungherese labda 'palla' (pp. 86-89) che presenta varie corrispondenze nelle lingue slave e balcaniche, oggetto di tante discussioni, come si può vedere anche dai più recenti dizionari etimologici. Egli, dopo ampio esame delle fonti archivistiche, propone per tale famiglia di parole una spiegazione interamente nuova e originale che riteniamo assai probabile, ma incompleta nella formulazione e giustificazione fonetica (sostanzialmente mancante). In questo breve articolo cercheremo di integrare le osservazioni di Hadrovics ch'egli ha esposto in forma, secondo noi, plausibile per quanto attiene i particolari della mutuazione.

L'opinione corrente relativa alla parola ungh. labda 'palla' (in vari sensi) è che si tratti di uno slavismo, mentre l'A., indicando altrove la vera fonte etimologica originaria, pensa esattamente il contrario e ritiene che sia stata, la voce magiara a diffondersu nell' area slava meridionale e balcanica in generale. Si può verificare l'ipotesi slava ad es. a partire dal dizionario etimologico del Bárczi del $1941^{3}$, ma anche prima come si desume dalla bibliografia citata dal medesimo autore; la forma ungh. labda (dal sec. XV) e ant. anche lapta (accanto a lapát 'pala') verrebbe dallo slavo, cfr. s.cr., slov. lopta 'palla' e anche 'specie di pala' (lopata) ecc. Ampie indicazioni fornisce inoltre Kniezsa nella monumentale opera del $1955^{4}$ sull' influsso slavo in ungherese, s.v. labda con i significati: 1. pila Ball, 2. globus, Kugel (a. 1493 e 1533 lapta). Il Kniezsa, secondo la tradizione, ritiene di poter risalire alla forma slava lopłta, cfr. il russo loptá, laptá 'pala del remo' ed anche 'racchetta' e unitamente il s. cr. lopta 'Ball', il kajkavo lopta 'pila', slov. lopta 'Spielballen' ecc. Secondo Kniezsa, 1. cit., il mutamento e il traslato di lopta 'pila' in labda 'golyó', ciò̀ 'pallottola', 'pallino' 5 sarebbe avvenuto in ungherese. Anche il TESz 6 ' $I$, p. 701 , s. v. Iabda, ripete in sostanza la medesima spiegazione con la citazione delle fonti tradizionali e i seguenti significati: 1. 'Spielball', 2. 'Kugel', 3. 'spielballähnlicher Gegenstand, Bestandteil' e 4. 'Ballschlegel' (la variante s. cr. kajk. labda 'palla' e slov. dial. labda sarebbe stata presa dall' ungherese). Credo che la fonte dell' equivoco (cosi pare anche a me), di collegare due famiglie di voci che hanno origine assai diversa, risalga al Berneker, SEWb. I, pp. 
732-3, ove sotto lopata 'pala', 'badile', 'remo', 'scapola' ecc., attestato da tante lingue slave a partire dall' a. bulg. (slavo eccl.) lopata 'TTúor' 'Wurfschaufel' (pala da grano), viene citato anche il russo loptá 'Schaufel, Ruderblatt' 'ein flaches Ding das am Ende breiter wird', 'ein Stock, eine Art Schaufel, Ruder mit dem man den Ball wirft' (cioè una specie di' racchetta'), 'Ballspiel', e cio che crea maggiore confusione anche il s.cr. lopta 'ball', slov. lopta idem, slovacco lopta 'idem' (dallo slavo verrebbe l' ungh. labda, lapta 'Ball'.. "schwerlich umgekehrt", come aveva sostenuto il Miklosich, Et. Wb. p. 174 "ohne das $r$. Wort zu beachten"). La voce lopata verrebbe, con Ablaut, da lapa 'palmo della mano', cfr. lit. lopeta 'Schaufel', lett. lapota 'Spaten', 'Schusterblatt', a. pruss. lopto 'Spaten' ecc. (v. anche per il lit. lopeta, Fraenkel, LEW I, 339-340 s.v. lãpas, ove è citata una ampia famiglia di parole tra cui lopeta). Anche il Vasmer, REWb. II, pp. 14-15, sotto russo laptá ripete le osservazioni del Berneker ("Magy. labda, lapta 'ball' ist entlehnt aus d. Slav, nicht umgekehrt") e ivi si aggiunge anche che sarebbe erronea la derivazione dal. ted. Latte , it. latta 'Schlag' "die für das $\underline{p}$ keine Erklärung bietet". Per completezza cito, oltre al Sadnik-Aitzetmüller, Handb. p. 263, nr. 475 (s.v. lopata ove si ripetono le annotazioni gia citate), anche il Pokorny, IEW, p. 679 che sotto le radici lëp-, lōp-, lop-' flach sein, Hand-, Fussflache, Schulterblatt, Schaufel, Ruderblatt', Iopa 'Hand, Pfote', elenca i numerosi derivati ie. tra cui il russo ecc. lapa 'Pfote, Tatze', abg. lopata 'Wurfschaufel', russo lopata 'Schaufel', lopatka 'Schulterblatt' (ecc.) e I'alb. lopetë 'Schaufel', uno slavismo. La forma ufficiale albanese, ora citata dal Fjalor i gjuhës shqupë p. 276, è lopatë -a (badile, vanga, remo); in romeno si conosce lopata 'pala' 'pala del remo' stavismo, ed anche lopta 'palla' che il Cioranescu, DERum. p. 485, deriva dall' ungh. lapta; si veda a questo proposito l'ampio lemma del Tamás, Etymologisch-historisches Wörterbuch der ungarischen Elemente im Rumänischen, Budapest 1966, p. 509 s.v. Ioptă 'Spielball' (il quale per la voce magiara ripete la nota bibliografia che ribadisce I'origine slava della parola).

Non minore confusione (a giudizio mio) appare nell' articolo lópata (s.cr., Vuk) dello Skok, ERHSJ II (1972), pp. 318-19 'pala', 'scapola' e vari richiami al traslato toponimico (anche in molti dialetti italiani la 'scapola' è derivata dal lat. pala, palòta, così anche in dial. ven. sett.). Lo Skok cita accanto a lopta anche lofta (Palmotić) $=$ lovta (Ljubiša) 'palla' etc. (si riconosce poi che la variante s.cr. - kajk. lapta è un prestito dall' ungherese). Nel Rječnik. VI (1904-1910) p. 155 s.v. lopta 'globus, pila, follis' ... si aggiunge "Misli se da je, kao i slovač. lopta od mág. labda, lapta ..".

Se accettiamo la brillante proposta di Hadrovics dobbiamo subito sottrarre alla famiglia di voci che fanno capo allo slavo lopata ecc. il magiaro labda, lapta (da cui proviene allora il s.cr. ecc. lopta) 'palla' (con varie accezioni) poiché quest'ultima parola avrebbe una storia interamente diversa e rappresenterebbe un prestito di origine romanza $o$, per esser più precisi, veneta. $E$ ' merito di H. aver attentamente esaminato $i$ contesti in cui compare labda 'palla' nei documenti antichi in latino, anche della Dalmazia, e di averne precisato $i$ significati. Egli sottolinea infatti, accanto al senso di 'palla da gioco', soprattutto 1' accezione militaresca che compare fin dal 1493 (fortezza di Jajce): "Pulveres 
pixidorum tria vasecula cum medio. Laptes septuagintas igneas decem et quattuor"; ad Eger a. 1508: "Laptha ignea magna decem et octo 18; Laptha ignea parva novem."; a. 1520 (ca.) Lapta wlgo twzes; 1528 (Gyula) "I ferreum instrumentum ad proiiciendum ignem vulgo lapta"; a. 1549 "Pila pro igne cum pice laptha"; a. 1554 (Gyula) "testaceae crematoriae vulgo zeneslapta 23", e seguono poi varie citazioni di passi in ungherese in cui si vede chiaramente come tale lapta si equivale a una "palla incendiaria" (tüzes lapta) una palla infuocata che si scagliava contro i nemici, una pallottola o simile. In testi paralleli veneto-dalmati compare al posto di labda, lapta un termine che ha tutta l'aria di essere perfettamente equivalente e precisamente bal(l)ot(t)a. Ritrascrivo alcuni passi riportati sempre dallo Hadrovics; da Zara a. 1351: "Item baliste XII a pexarola cum pexarolis et sagitamentum etiam pro dictis balistis in ea quantitate, que fuerit opportunum. Item sclopi VIII, cum quibus prohici possint sagitamenta et balote cum igne" (MonslavMerid. 3, 205); è chiaro come osserva lo H. che balotas cum igne si equivale perfettamente a tüzes laptak sopra menzionato ('palle incendiarie'). All'a. 1528 sempre da doc. zaratino: "Vna balota de fero de spingarda". Nel Du Cange ballota è fatta equivalere a 'pilula seu glans ferrea vel plumbea' ecc. Aggiungo qui un brevissimo campionario lat. mediev. tratto da Sella, Gloss. lat. -it. p. 53 s.v. balota 'palla, pallottola', a Pola sec. XIV; "ballotis a sclopo de ferro et plumbo" a Belluno a. 1379; balotam ferream aut plumbeam" ad Adria a. 1402; inoltre "Cerebotanis utebantur, lapides seu balottas de girla (cioè 'creta') ... proicientes", Udine a. 1425 (Sella cit. p. 148), ecc.

Dal Battaglia, GDLI-UTET II, 18 traggo le seguenti informazioni: ballotta 3 , ant. proiettile; piccola palla che si lancia con la balestra (più per la caccia che per combattimento [ma ciò vale per gli esempi italiani riportati], con le segg. citazioni da Folgore di S. Gimignano (XIII sec.) "Di settembre vi do diletti tanti ... .. bolz'e balestre dritt'e ben portanti/archi, strati, ballotte e ballottieri"; e da Leonardo da Vinci "L'aria sia piena di saettume di diverse regioni: chi monti, chi discende, quali sia per linea piana: e le ballotte delli scoppietti sieno accompagnate d'alquanto fumo dirieto al lor corso". Si tratta di voce di origine italiana settentrionale ( $\mathrm{V}$. DEI I, 417 e Prati, VEI 95 il quale dichiara espressamente I'origine veneta di ballotta, ballottare, mentre ballottaggio verrebbe dal francese), e il Boerio ${ }^{3}$ p. 59 , cita belota, oltre che nel senso di 'pallottola, voto (piccola palla di cenci o altro con cui si raccolgono i suffragi degli squittinii)' anche 'pallottola di terracotta' e.v. anche sotto balotina "... ad uccidere gli smerghi colle pallottole di terra cotta detta volgarmente balote ch'essi tirano colla balestra". Si tratta di un derivato di bal(l)a col suffisso -ŏttus, voce di origine francone o ant. alto tedesca: balla (REW 908, 1, Gamillscheg, Rom. Germ. I, p. 248). Quanto al suffisso il Rohlfs, Gramm. storica della lingua italiana ... III (1969), p. 1551146 -otto, attribuisce a tale variante di -ittus un fondamentale valore diminutivo che peraltro in dialetti it. settentrionali si trasforma spesso in accrescitivo. Lo Hadrovics sottolinea giustamente gli analoghi contesti e le perfette corrispondenze di significato nei testi lat. mediev. e ungheresi tra ballotta (e varianti tra cui anche balocta) e ungh. Iapta, labda "palla incendiaria" di ferro o di terracotta. Egli propone di spiegare la voce ungherese - è il primo per quanto mi consta ad aver avuto tale intuizione-e slavo-balcanicacome prestito dalla succitata parola veneta. $\mathrm{Ma}$, a questo punto, non mancano le difficoltà di 
ordine fonetico ch' egli cerca solo in parte di superare. Lo H. osserva ad es., che nei prestiti da lingue neolatine in ungherese sia rimasta intatta soprattutto la parte della parola dopo l'accento e che pertanto siano molto comuni le aferesi sillabiche (egli cita forme piuttosto banali quali Alessandro > Sandro e piu tipico per il s.cr. a Zara Anastasia divenuta la Sveta (santa) Stošija patrona della città). $\mathrm{Ma}$ anche pensando a $\mathrm{d}$ un $\overline{\mathrm{b}}$ lota ridotto a lota, le difficoltà permangono ed esse sono gravi per qualsiasi assenza di traccia della labiale. Egli stesso osserva che sarebbe seducente (《 csábitó volna 》) poter partire da balocta poiché -ct- spesso si trasforma in -pt- in ungherese come insegnano esempi quali iktat $\overline{\rangle}$ iptat, hektika > heptika, röktön > röptön ecc. Ma egli si rende anche conto che tali forme con -ct- erano soltanto grafiche, devute a false ricostruzioni degli scribi e che alcuna persona avrà mai pronunciato balocta. In sostanza lo $\mathrm{H}$. si accontenta di aver presentato un quadro esauriente e convincente delle equivalenze veneto-ungheresi e di aver trovato in certo senso una spiegazione plausibile per labda, lapta (onde le forme slave). Sono i documenti esibiti e l'ambiente culturale che essi evocano a confermare la sua supposizione. Ma penso si possa giungere ad una interpretazione ancor più persuasiva qualora si risolva correttamente l'aporia fonetica. Secondo noi è infatti possibile ricorrere ad una metatesi con successiva perdita, non tanto insolita, della vocale mediana postonica. Ritengo che la metatesi, abbastanza antica (prima del sec. XV) in area danubiana sia stata favorita anche dalla presenza dell'articolo la, e mi immagino pertanto la seguente trafila: la balota > la labota e con ritrazione d'accento sulla prima sillaba tipica del magiaro- (la) làbota e successivamente lab(o)ta, con assimilazione poi lapta o labda. I fenomeni di metatesi sono molto comuni anche in ungherese e mi basterebbe rinviare, per gli esempi, ad un capitoletto della nota opera A magyar nyelv története 7 . Ma analoghe trasformazioni sono frequenti anche in prestiti dall'italiano, e mi basterebbe citare l'esempio di mazsola da ant malozsa ciò it. malvasia, studiato minuziosamente da L. Hadrovics, Jövev. cit. pp. 106-108, oppure cfr. kandalló 'camino'8 da caldano (se tale etimo è corretto), ammesso anche dal TESz II, 343, ecc. Quanto alla caduta di vocali interne nei prestiti potrei citare un ampio campionario specie per -e- ed -i- (basti ricordare l'ungh. kámzsa dal lat. camisia o meglio dal veneto kamiźà; TESz II, 338). Si noti anche csáklya se viene da zagaglia, come è stato supposto anche da Hadrovics, Jövev. cit. p. 96. Ma si può indicare il medesimo fenomeno come già in atto nella storia dell' ungherese a partire dal 1000 , per cui mi basti rinviare al volume di L. Benkő e Samu Imre, The Hungarian Language, Budapest 1972, p. 65 (si ricordi ad es. ant. urusag $>$ orszag 'stato', holovan $>$ halvany 'pallido', bukurut, bukrut > bokrot 'cespuglio', ecc.

Mi pare comunque che l'ipotesi di Hadrovics, da me perfezionata, sia preferibile a quelle sinora prospettate e che peccano a causa di un evidente fraintendimento. Tale abbaglio sarebbe assai simile a quello commesso da chi mettesse insieme, per il rispetto etimologico, l'it. palla (s. cr. lopta) con pala (s. cr. lopata) unicamente a causa della quasi perfetta coincidenza fonetica (e in una pronuncia it. sett. palla si equivale quasi a pala) e per una falsa interpretazione, veramente ingannevole, di racchetta (cfr. russo loptà o slavo in genere lopata) che casualmente sta in rapporto complementare, nel gioco, con la palla (anche in dialetti veneti la palota può essere equivalente a 'racchetta' e a 'scapola'). 
1 Edito in "Nyelvtudományi értekezések" [Dissertazioni linguistiche] nr. 50, Akadémiai kiadó, Budapest 1965 (di pp. 115), ove l'A. discute con ricerche d'archivio nuove e con proposte etimologiche originali, di cinquantasei parole ungheresi di cui 25 si riferiscono ad elementi slavi, 13 sarebbero prestito dal tedesco e 19 dall'italiano. Sull'opera dello Hadrovics si veda anche l'ampia rassegna critica di Miklós FOGARASI, Risultati ed insegnamenti di un metodo complesso nelle ricerche etimologiche, in AION-Ling. VIII (1968), pp. 187-205; $\mathrm{v}$. anche la mia comunicazione patavina Postille alle voci di origine italiana riportate dal TESz, in "Atti del Convegno linguistico italo-ungherese", 27-29 ottobre 1976 (in corso di stampa).

2 In "Nyelvtud. értekezések" cit. nr. 88, Budapest 1975 (di pp. 135); tale studio 'e suddiviso, oltre all'introduzione, alla bibliografia e all'indice delle parole, in otto capitoli di cui il IV riguarda i prestiti dallo slavo, il $\mathrm{V}$ dall'italiano e il VI dal tedesco.

3 Géza BÁRCZI, Magyar szófejtó szótar, Budapest 1941, p. 181.

4 Istvan KNIEZSA, A magyar nyelv szláv jövevényszavai, Budapest 1955, I, pp. 298-99.

5 Osservo per inciso che golyó 'palla' è un prestito dall'italiano e precisamente da "coglione", nella forma settentrionale koió(n), forse diffusa da Venezia e attraverso una variante senza $-\underline{n}$ comune ad es. al bergamasco (i Bergamaschi erano numerosi a Venezia).

6 A magyar nyelv történeti-etimológiai szótára (diretto da Loránd BENKơ), II, Akadémiai kiadó, Budapest 1970 (in questi giorni è uscito il terzo volume conclusivo della fondamentale opera; pare che sarà seguito da un supplemento).

7 BÁRCZI Géza-BENKO์ Loránd-BERRÁR Jolán, A magyar nyelv története, Budapest 1967, pp. 128-129.

8 Per i prestiti italiani in ungherese è sempre comodo il panorama generale offertoci da Ferenc KARÁNTHY, Olasz jövevényszavaink [i nostri prestiti italiani], Budapest 1947 (e si veda anche la mia comunicazione citata alla nota 1).

Povzetek

\section{BENEŠKO BALOTA: IZPOSOJENKA V BALKANSKO-PODONAVSKI SFERI}

Avtor predstavlja leksikalne študije madžarskega jezikoslovca Lászla Hadrovicsa in ocenjuje nekatere njegove etimološke razlage, ki predpostavljajo izposojanje iz italijanščine. Zaustavlja se pri besedi labda 'žoga'; na splošno velja beseda za izposojenko iz slovanskih jezikov, Hadrovics pa misli, da je ravno narobe: madžarska beseda naj bi se razŠirila $\mathrm{v}$ besednjak nekaterih slovanskih jezikov (lopta je izpričano tudi na slovenskih tleh). Avtor sprejema Hadrovicsevo mnenje, da je labda iz beneškega balota, kljub težavam, ki jih predstavlja glasovna oblika. Ker pa je balota dokumentirana $\mathrm{v}$ beneških zapisih $\mathrm{v}$ Dalmaciji $\mathrm{v}$ pomenu 'žoga' in ker je metateza pri izposojenkah čest pojav (na prim. it. malvasia : madž. malozsa, danes mazsola), se zdi Hadrovicseva hipoteza sprejemljiva. 